\title{
Resolving the Puzzle of the Pion-Photon Transition Form Factor
}

\section{Wolfgang Lucha}

Institute for High Energy Physics, Austrian Academy of Sciences, Nikolsdorfergasse 18, A-1050 Vienna, Austria

E-mail: Wolfgang.Lucha@oeaw.ac.at

\section{Dmitri Melikhov*}

Institute for High Energy Physics, Austrian Academy of Sciences, Nikolsdorfergasse 18, A-1050

Vienna, Austria,

Faculty of Physics, University of Vienna, Boltzmanngasse 5, A-1090 Vienna, Austria, and

D. V. Skobeltsyn Institute of Nuclear Physics, Moscow State University, 119991, Moscow, Russia

E-mail: dmitri_melikhov@gmx.de

\begin{abstract}
We investigate the form factors for pseudoscalar-meson-photon transitions by means of dispersive QCD sum rules and demonstrate that most of the measurements done so far (in particular, those by BABAR for $\eta, \eta^{\prime}$ and $\eta_{c}$ and by Belle for $\pi^{0}$ ) are, on the one hand, compatible with each other and with the saturation required by factorization theorems obtained from perturbative $\mathrm{QCD}$ and, on the other hand, give a hint that saturation is effective already at relatively low momentum transfers $Q^{2}$; this hypothesis is supported by experimental data for the charged-pion elastic form factor available in the range $Q^{2} \approx 2-4 \mathrm{GeV}^{2}$. The only exception are the BABAR results for the $\pi^{0} \gamma$ transition form factor, which do not fit into such picture. We point out that results expected from SHMS at JLab on the pion elastic form factor in the region $Q^{2} \approx 5-8 \mathrm{GeV}^{2}$ will provide the ultimate test of saturation and factorization and strongly impact our general view of the form factors up to infinitely large $Q^{2}$.
\end{abstract}

The European Physical Society Conference on High Energy Physics - EPS-HEP2013

18-24 July 2013

Stockholm, Sweden

\footnotetext{
${ }^{*}$ Speaker.
} 


\section{Introduction: Two-Photon Fusion to Pseudoscalar Mesons}

Transitions $\gamma^{*} \gamma^{*} \rightarrow P$ of two virtual photons $\gamma^{*}$ into some pseudoscalar meson $P=\pi^{0}, \eta, \eta^{\prime}, \eta_{c}$ are processes that provide important tests of the dynamics of exclusive processes in QCD. Recently, a large amount of experimental data on this kind of reaction has been gathered [1 -5]. The transition amplitude enjoys a particularly simple structure and involves only a single form factor, $F_{P \gamma \gamma}\left(q_{1}^{2}, q_{2}^{2}\right)$ :

$$
\left\langle\gamma^{*}\left(q_{1}\right) \gamma^{*}\left(q_{2}\right) \mid P(p)\right\rangle=\mathrm{i} \varepsilon_{\varepsilon_{1} \varepsilon_{2} q_{1} q_{2}} F_{P \gamma \gamma}\left(q_{1}^{2}, q_{2}^{2}\right) .
$$

The behaviour of the form factor $F_{P \gamma \gamma}\left(q_{1}^{2}, q_{2}^{2}\right)$ at asymptotically large spacelike momentum transfers $q_{1}^{2} \equiv-Q_{1}^{2} \leq 0, q_{2}^{2} \equiv-Q_{2}^{2} \leq 0$ results from a QCD factorization theorem by Lepage and Brodsky [6]:

$$
F_{P \gamma \gamma}\left(Q_{1}^{2}, Q_{2}^{2}\right) \underset{Q_{1,2}^{2} \rightarrow \infty}{\longrightarrow} 12 e^{2} f_{P} \int_{0}^{1} \frac{\mathrm{d} \xi \xi(1-\xi)}{Q_{1}^{2} \xi+Q_{2}^{2}(1-\xi)} .
$$

The kinematical situation relevant to experiment is characterized by an almost on-shell $\left(Q_{1}^{2} \approx 0\right)$ and an off-shell $\left(Q^{2} \equiv Q_{2}^{2} \geq 0\right)$ photon: In this case, the pion-photon transition form factor depends only on the variable $Q^{2} \equiv Q_{2}^{2}$ and will be labeled by $F_{\pi \gamma}\left(Q^{2}\right)$. For large $Q^{2}$, it behaves asymptotically like

$$
Q^{2} F_{\pi \gamma}\left(Q^{2}\right) \underset{Q^{2} \rightarrow \infty}{\longrightarrow} \sqrt{2} f_{\pi}, \quad f_{\pi}=0.130 \mathrm{GeV} .
$$

Upon taking into account meson mixing effects, similar relations arise also for the $\eta$ and $\eta^{\prime}$ mesons.

\section{Dispersive QCD Sum Rules for the $\gamma^{*} \gamma^{*} \rightarrow P$ Transition Form Factor}

Let us base our QCD sum-rule analysis of the $\gamma^{*} \gamma^{*} \rightarrow P$ transition form factor on the amplitude

$$
\left\langle 0\left|j_{\mu}^{5}\right| \gamma^{*}\left(q_{1}\right) \gamma^{*}\left(q_{2}\right)\right\rangle=e^{2} T_{\mu \alpha \beta}\left(p \mid q_{1}, q_{2}\right) \varepsilon_{1}^{\alpha} \varepsilon_{2}^{\beta}, \quad p \equiv q_{1}+q_{2},
$$

where $j_{\mu}^{5}$ is the axial-vector quark current and $\varepsilon_{1,2}$ are the polarization vectors of the photons. Out of the four independent Lorentz structures of this amplitude (cf. Refs. [7, 8]), here only one is relevant:

$$
T_{\mu \alpha \beta}\left(p \mid q_{1}, q_{2}\right)=p_{\mu} \varepsilon_{\alpha \beta q_{1} q_{2}} \mathrm{i} F\left(p^{2}, Q_{1}^{2}, Q_{2}^{2}\right)+\cdots .
$$

The invariant amplitude $F\left(p^{2}, Q_{1}^{2}, Q_{2}^{2}\right)$ can be written as dispersion integral over $p^{2}$ (at fixed $Q_{1}^{2}, Q_{2}^{2}$ )

$$
F\left(p^{2}, Q_{1}^{2}, Q_{2}^{2}\right)=\frac{1}{\pi} \int_{s_{\mathrm{th}}}^{\infty} \frac{\mathrm{d} s}{s-p^{2}} \Delta\left(s, Q_{1}^{2}, Q_{2}^{2}\right)
$$

of the physical spectral density $\Delta\left(s, Q_{1}^{2}, Q_{2}^{2}\right)$, with lower endpoint fixed by the physical threshold $s_{\text {th }}$. Perturbative QCD provides the spectral density as an expansion in powers of the strong coupling $\alpha_{\mathrm{s}}$ :

$\Delta_{\mathrm{pQCD}}\left(s, Q_{1}^{2}, Q_{2}^{2} \mid m\right)=\Delta_{\mathrm{pQCD}}^{(0)}\left(s, Q_{1}^{2}, Q_{2}^{2} \mid m\right)+\frac{\alpha_{\mathrm{s}}}{\pi} \Delta_{\mathrm{pQCD}}^{(1)}\left(s, Q_{1}^{2}, Q_{2}^{2} \mid m\right)+\frac{\alpha_{\mathrm{s}}^{2}}{\pi^{2}} \Delta_{\mathrm{pQCD}}^{(2)}\left(s, Q_{1}^{2}, Q_{2}^{2} \mid m\right)+\cdots$

also involves the mass $m$ of the quark propagating in the loop spanned by $j_{\mu}^{5}$ and the photon vertices. This 1-loop triangle diagram, with $j_{\mu}^{5}$ and two electromagnetic currents at its vertices, contributes to $\Delta_{\mathrm{pQCD}}\left(s, Q_{1}^{2}, Q_{2}^{2} \mid m\right)$ the lowest-order term $\Delta_{\mathrm{pQCD}}^{(0)}\left(s, Q_{1}^{2}, Q_{2}^{2} \mid m\right)$ [9]; the 2-loop $O\left(\alpha_{\mathrm{s}}\right)$ term, due to the exchange of a gluon between two quark legs, vanishes [10]; the 3-loop $O\left(\alpha_{\mathrm{s}}^{2}\right)$ term is non-zero [11]. 
For low $s$ values, the physical spectral density differs from the perturbative $\Delta_{\mathrm{pQCD}}\left(s, Q_{1}^{2}, Q_{2}^{2} \mid m\right)$ as the former involves meson pole and hadron continuum. In the $I=1$ channel, for instance, it reads

$$
\Delta\left(s, Q_{1}^{2}, Q_{2}^{2}\right)=\pi \delta\left(s-m_{\pi}^{2}\right) \sqrt{2} f_{\pi} F_{\pi \gamma \gamma}\left(Q_{1}^{2}, Q_{2}^{2}\right)+\theta\left(s-s_{\mathrm{th}}\right) \Delta_{\mathrm{cont}}^{(I=1)}\left(s, Q_{1}^{2}, Q_{2}^{2}\right) .
$$

$Q C D$ sum rules enable us to construct relations between properties of ground-state hadrons and spectral densities of QCD correlation functions by equating QCD- and hadron-level representations of $F\left(p^{2}, Q_{1}^{2}, Q_{2}^{2}\right)$, performing a Borel transformation $p^{2} \rightarrow \tau$ to a new "Borel" variable $\tau$ to suppress the hadron continuum, and implementing quark-hadron duality by introducing a low-energy cut on the spectral representation $[12,13]$. Potentially dangerous nonperturbative power corrections rising with $Q_{1,2}^{2}$ are absent in the local-duality (LD) limit $\tau=0$ [14]. The procedure gives for $F_{P \gamma \gamma}\left(Q_{1}^{2}, Q_{2}^{2}\right)$

$$
\pi f_{P} F_{P \gamma \gamma}\left(Q_{1}^{2}, Q_{2}^{2}\right)=\int_{4 m^{2}}^{s_{\mathrm{eff}}\left(Q_{1}^{2}, Q_{2}^{2}\right)} \mathrm{d} s \Delta_{\mathrm{pQCD}}\left(s, Q_{1}^{2}, Q_{2}^{2} \mid m\right),
$$

with all details of nonperturbative-QCD dynamics encoded in some effective threshold $s_{\text {eff }}\left(Q_{1}^{2}, Q_{2}^{2}\right)$. The actual challenge in this game is to formulate reliable criteria for fixing effective thresholds [12].

For notational simplicity, from now on we switch to variables $Q^{2}$ and $\beta$ defined by $Q^{2} \equiv Q_{2}^{2}$ and $0 \leq \beta \equiv Q_{1}^{2} / Q_{2}^{2} \leq 1$. For given $\beta$, our effective threshold $s_{\text {eff }}\left(Q^{2}, \beta\right)$ in the limit $Q^{2} \rightarrow \infty$ is found by matching to the asymptotic factorization result. In the general case $m \neq 0$, this gives $s_{\text {eff }}\left(Q^{2} \rightarrow \infty, \beta\right)$ as a function of $\beta$. Only for massless fermions, $m=0$, the asymptotic value is reproduced for all $\beta$ if $s_{\text {eff }}\left(Q^{2}, \beta\right)$ behaves like $s_{\text {eff }}\left(Q^{2} \rightarrow \infty, \beta\right)=4 \pi^{2} f_{\pi}^{2}$. The LD model assumes that also for finite values of $Q^{2}, s_{\text {eff }}\left(Q^{2}, \beta\right)$ is tolerably approximated by the $Q^{2} \rightarrow \infty$ limit, i.e., $s_{\text {eff }}\left(Q^{2}, \beta\right)=s_{\text {eff }}\left(Q^{2} \rightarrow \infty, \beta\right)$. Employing the abbreviation $F_{P \gamma}\left(Q^{2}\right) \equiv F_{P \gamma \gamma}\left(0, Q^{2}\right)$ for the "empirical" pseudoscalar-meson-photon transition form factor, its LD expression for $Q_{1}^{2}=0$ and $m=0$ is given, in the single-flavour case, by

$$
F_{P \gamma}\left(Q^{2}\right)=\frac{1}{2 \pi^{2} f_{P}} \frac{s_{\mathrm{eff}}\left(Q^{2}\right)}{S_{\mathrm{eff}}\left(Q^{2}\right)+Q^{2}} .
$$

Irrespective of the behaviour of $s_{\text {eff }}\left(Q^{2}\right)$ for $Q^{2} \rightarrow 0, F_{P \gamma}\left(Q^{2}=0\right)$ is related to the axial anomaly [7].

\section{Local-Duality Approach to Pseudoscalar-Meson-Photon Transition Form Factors}

Let us discuss the various mesons in the order of increasing amount of troubles to be overcome.

\subsection{Form Factor for the $\eta_{c}$-Meson-Photon Transition $\gamma^{*} \gamma^{*} \rightarrow \eta_{c}$}

For massive quarks, we may study the correlators $\langle A V V\rangle$ and $\langle P V V\rangle$ of two vector currents and either an axial-vector or a pseudoscalar quark current, respectively, in order to find LD sum rules for the transition form factor under consideration [8]. By satisfying the perturbative-QCD factorization theorem, the asymptotic values $s_{\text {eff }}\left(Q^{2} \rightarrow \infty, \beta\right)$ for both correlators are derived. The exact effective thresholds valid for $\langle A V V\rangle$ and $\langle P V V\rangle$ prove to differ from each other [8]. Under the LD hypothesis we obtain the results depicted in Fig. 1. Clearly, for very small $Q^{2}$ the LD model is, by construction, not applicable. Adopting it, nevertheless, the full way down to $Q^{2}=0$ gives $F_{\eta_{c} \gamma}(0)=0.067 \mathrm{GeV}^{-1}$ from $\langle A V V\rangle$ and $F_{\eta_{c} \gamma}(0)=0.086 \mathrm{GeV}^{-1}$ from $\langle P V V\rangle$. These outcomes must be confronted with the corresponding experimental data $F_{\eta_{c} \gamma}(0)=(0.08 \pm 0.01) \mathrm{GeV}^{-1}$ : For both correlators used, the LD model performs reasonably well over a broad $Q^{2}$ range starting at surprisingly low $Q^{2}$; cf. Ref. [15]. 


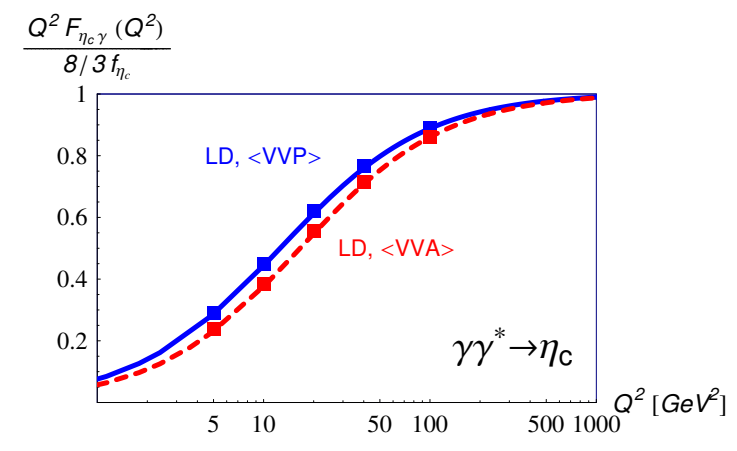

(a)

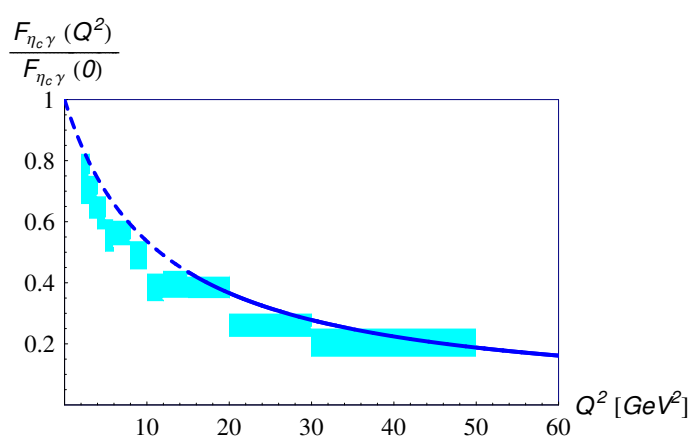

(b)

Figure 1: Form factor for the transition $\gamma \gamma^{*} \rightarrow \eta_{c}$ : (a) form factor for finite $Q^{2}$, arising from LD sum rules for $\langle A V V\rangle$ and $\langle P V V\rangle$; (b) LD model for the correlator $\langle P V V\rangle$ confronted with experimental data by BABAR [3].

\subsection{Form Factors for the $\eta^{(\prime)}$-Meson-Photon Transitions $\gamma \gamma^{*} \rightarrow\left(\eta, \eta^{\prime}\right)$}

A minor complication arises in any description of two-photon fusion to $\eta$ and $\eta^{\prime}$; the mixing of their nonstrange $n \sim(\bar{u} u+\bar{d} d) / \sqrt{2}$ and strange $s \sim \bar{s} s$ components has to be taken into account [16]:

$$
F_{\eta \gamma}\left(Q^{2}\right)=F_{n \gamma}\left(Q^{2}\right) \cos \phi-F_{s \gamma}\left(Q^{2}\right) \sin \phi, \quad F_{\eta^{\prime} \gamma}\left(Q^{2}\right)=F_{n \gamma}\left(Q^{2}\right) \sin \phi+F_{s \gamma}\left(Q^{2}\right) \cos \phi,
$$

with mixing angle $\phi \approx 38^{\circ}$. The LD predictions for the flavour form factors $F_{n \gamma}\left(Q^{2}\right)$ and $F_{s \gamma}\left(Q^{2}\right)$ are

$$
f_{n} F_{n \gamma}\left(Q^{2}\right)=\int_{0}^{s_{\text {eff }}^{(n)}\left(Q^{2}\right)} \mathrm{d} s \Delta_{n}\left(s, Q^{2}\right), \quad f_{s} F_{s \gamma}\left(Q^{2}\right)=\int_{0}^{s_{\text {eff }}^{(s)}\left(Q^{2}\right)} \mathrm{d} s \Delta_{s}\left(s, Q^{2}\right) .
$$

The two separate effective thresholds $s_{\text {eff }}^{(n)}\left(Q^{2}\right)$ and $s_{\text {eff }}^{(s)}\left(Q^{2}\right)$ need not be identical: $s_{\text {eff }}^{(n)}\left(Q^{2}\right)=4 \pi^{2} f_{n}^{2}$ and $s_{\text {eff }}^{(s)}\left(Q^{2}\right)=4 \pi^{2} f_{s}^{2}$, from the LD point of view, with $f_{n} \approx 1.07 f_{\pi}$ and $f_{s} \approx 1.36 f_{\pi}$. The outcomes of this LD model [7, 8] show satisfactory agreement with available experimental data [1, 4] (Fig. 2).

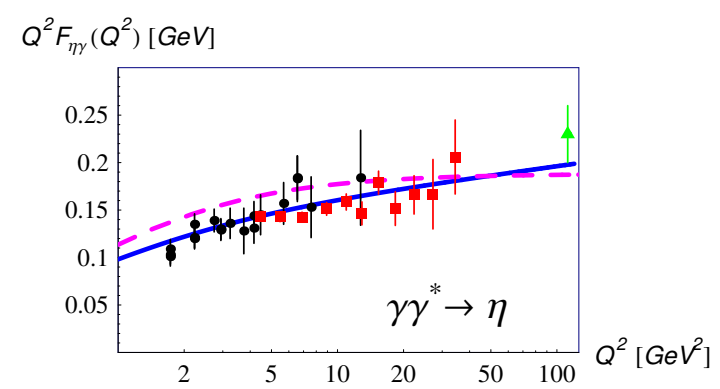

(a)

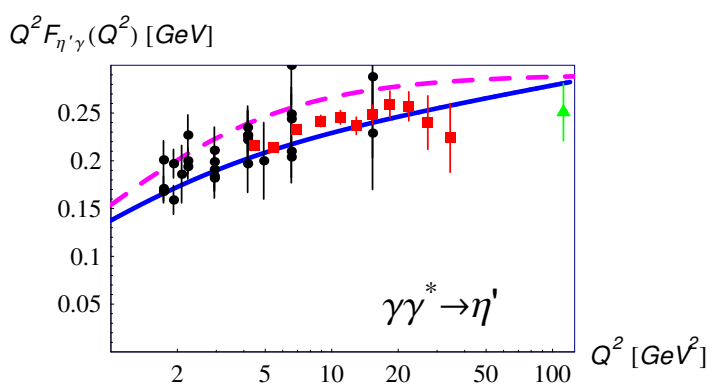

(b)

Figure 2: Form factors $F_{\left(\eta, \eta^{\prime}\right) \gamma}\left(Q^{2}\right)$ for the transitions $\gamma \gamma^{*} \rightarrow\left(\eta, \eta^{\prime}\right)$ : LD predictions [7, 8] (dashed magenta lines) and recent fits [17] (solid blue lines) to the experimental data [1, 4] for $\gamma \gamma^{*} \rightarrow \eta$ (a) and $\gamma \gamma^{*} \rightarrow \eta^{\prime}$ (b). 


\subsection{Form Factor for the Neutral-Pion-Photon Transition $\gamma \gamma^{*} \rightarrow \pi^{0}$}

The current situation with the two-photon fusion to the neutral pion $\pi^{0}$ constitutes a true source of worry, even on rather general grounds: The behaviour of each of the $\eta, \eta^{\prime}$, and $\pi^{0}$ transition form factors for large $Q^{2}$ is described by spectral densities computed from perturbative-QCD diagrams; it therefore should be identical for all light pseudoscalar mesons [17]. This fact becomes evident upon noting that the $\langle A V V\rangle \mathrm{QCD}$ sum rule in its LD limit $\tau=0$ is equivalent to the anomaly sum rule [18]

$$
2 \sqrt{2} \pi^{2} f_{\pi} F_{\pi \gamma}\left(Q^{2}\right)=1-2 \pi \int_{s_{\mathrm{th}}}^{\infty} \mathrm{d} s \Delta_{\text {cont }}^{(I=1)}\left(s, Q^{2}\right) .
$$

The same relations hold, mutatis mutandis, also for the $I=0$ and $\bar{s}$ channels. Thus, the form factors $F_{\pi \gamma}\left(Q^{2}\right), F_{\eta \gamma}\left(Q^{2}\right)$, and $F_{\eta^{\prime} \gamma}\left(Q^{2}\right)$ at large $Q^{2}$ are controlled by the behaviour of the respective hadron continuum contributions $\Delta_{\text {cont }}\left(s, Q^{2}\right)$ for large $s$ [17]. Quark-hadron duality assures us that the latter must equal their QCD-level counterparts $\Delta_{\mathrm{pQCD}}\left(s, Q^{2}\right)$, which, as purely perturbative quantities, are identical for all channels. Surprisingly, BABAR reports in the case of the pion transition form factor a distinct disagreement both with the $\eta$ and $\eta^{\prime}$ form factors and with the LD predictions for $Q^{2}$ up to $Q^{2} \approx 40 \mathrm{GeV}^{2}$. Still worse, in contrast to our quantum-mechanical expectations the deviations from LD predictions rise with $Q^{2}$ even in the region $Q^{2} \approx 40 \mathrm{GeV}^{2}$. So, QCD has a hard time when trying to understand the BABAR $\pi^{0}$ results (cf. also Ref. [19]). In this context, a recent Belle measurement brought a great relief to theory since, although statistically consistent with the BABAR data [20,21], the Belle findings for the $\pi^{0} \gamma$ transition form factor are fully compatible with existing $\eta$ and $\eta^{\prime}$ data as well as with the very likely onset of the $\mathrm{LD}$ regime already in the range $Q^{2} \gtrsim 5-10 \mathrm{GeV}^{2}$ (Fig. 3).

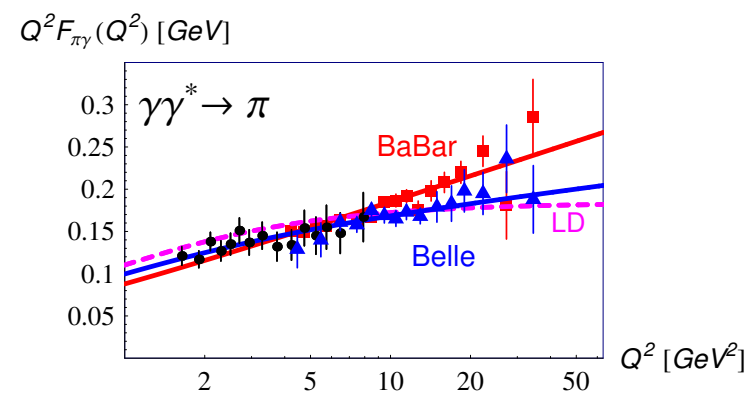

(a)

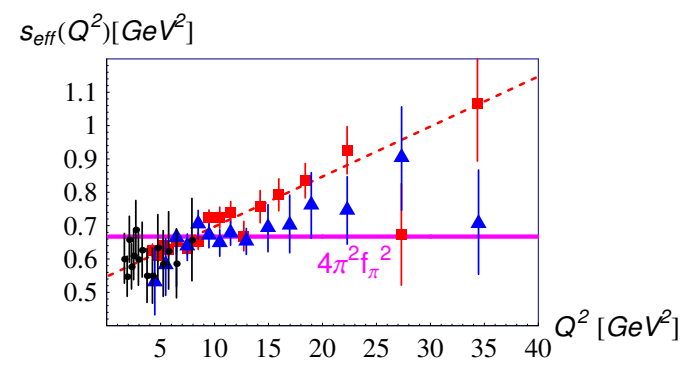

(b)

Figure 3: LD approach (magenta lines) to the transition $\gamma \gamma^{*} \rightarrow \pi^{0}$ : (a) form factor $F_{\pi \gamma}\left(Q^{2}\right)$ vs. fits [17] (solid lines) to experiment $[1,2,5]$; (b) equivalent effective threshold $s_{\text {eff }}\left(Q^{2}\right)$, fixed for each data point by Eq. (2.1).

\section{Elastic Form Factor of the Charged Pion}

Our recent detailed reanalysis of the charged pion's elastic form factor within the framework of QCD sum rules in LD limit [7] lends strong support to this LD concept: the exact effective threshold for this case may be computed from precision measurements at low $Q^{2}$ (Fig. 4). Taking into account 
- the experimental results for the effective threshold $s_{\text {eff }}\left(Q^{2}\right)$ at low momentum transfer $Q^{2}$ and

- its general feature of converging to the asymptotic value $4 \pi^{2} f_{\pi}^{2}$ in the region $Q^{2} \gtrsim 4-6 \mathrm{GeV}^{2}$, there are good reasons to expect that $s_{\text {eff }}\left(Q^{2}\right)$ for the pion's elastic form factor reaches its asymptotic value already somewhere near $Q^{2} \approx 4-6 \mathrm{GeV}^{2}$. If so, the $\mathrm{LD}$ approach predicts the pion elastic form factor accurately for all $Q^{2} \gtrsim 4-6 \mathrm{GeV}^{2}$; forthcoming measurements by JLab's SHMS will tell [22].

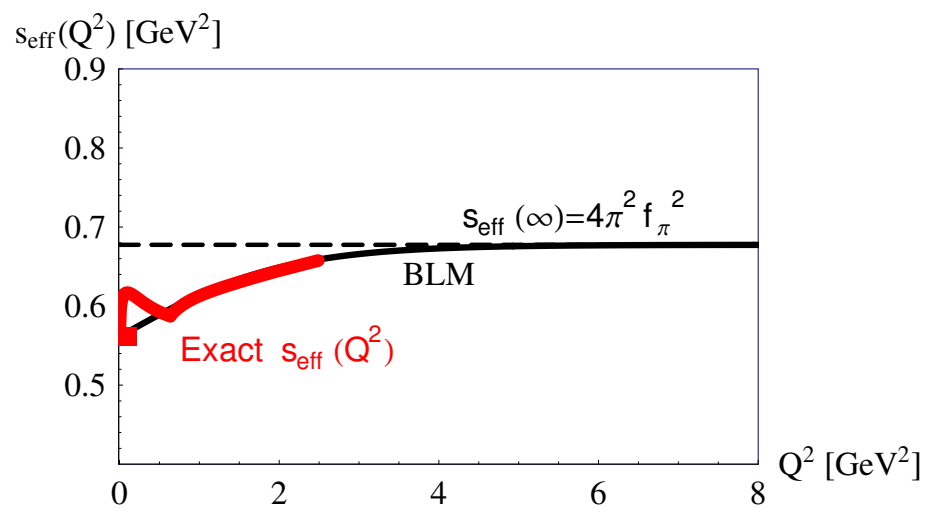

Figure 4: Effective threshold $s_{\text {eff }}\left(Q^{2}\right)$ for the charged-pion elastic form factor: exact behaviour as function of momentum transfer derived from experiment (red line) vs. a simple parametrization labeled BLM by Braguta et al. (solid black line) [7] that approaches its expected asymptotic limit $s_{\mathrm{eff}}(\infty)=4 \pi^{2} f_{\pi}^{2}$ (dashed black line).

\section{Summary of Main Results and Conclusions}

Our improved QCD sum-rule approach promotes the effective continuum threshold entering in the QCD-level spectral representation of the correlator under consideration to a truly key quantity of the modified formalism. Determining this object by matching the predictions of our technique to the consequences of QCD factorization theorems, we studied the transition form factors for two-photon fusion to the $\pi^{0}, \eta, \eta^{\prime}$, and $\eta_{c}$ mesons by QCD sum rules in LD limit, and arrived at crucial insights.

- For momentum transfer $Q^{2}$ larger than a few $\mathrm{GeV}^{2}$, this $\mathrm{LD}$ model is expected to reproduce to a satisfactory degree the transition form factors for all $P \rightarrow \gamma \gamma^{*}$ processes: for $P=\eta, \eta^{\prime}, \eta_{c}$, it indeed does; for $P=\pi^{0}$, BABAR [2] requires an LD-violating linear rise of $s_{\text {eff }}\left(Q^{2}\right)$ instead of its approach to a constant [Fig. 3(b)] but, by confirming the LD claim, Belle [5] saves our day.

- Assuming applicability of the LD ideas also to the elastic form factor of the charged pion, our findings lead us to suspect that in this case the accuracy of the LD model increases with $Q^{2}$ in the region $Q^{2} \approx 4-8 \mathrm{GeV}^{2}$ [7]. Accurate experimental data on the $\pi^{ \pm}$form factor suggest that the $\mathrm{LD}$ value $s_{\mathrm{eff}}(\infty)=4 \pi^{2} f_{\pi}^{2}$ of the effective threshold is reached already at $Q^{2} \approx 4-8 \mathrm{GeV}^{2}$; this claim looks forward to its confrontation with the results by the JLab upgrade SHMS [22].

Acknowledgments. D.M. thanks B. Stech, S. Brodsky, A. Oganesian and O. Teryaev for numerous helpful discussions. D.M. was supported by the Austrian Science Fund (FWF), Project No. P22843. 


\section{References}

[1] H. J. Behrend et al., Z. Phys. C 49 (1991) 401; J. Gronberg et al., Phys. Rev. D 57 (1998) 33.

[2] B. Aubert et al., Phys. Rev. D 80 (2009) 052002.

[3] J. P. Lees et al., Phys. Rev. D 81 (2010) 052010.

[4] P. del Amo Sanchez et al., Phys. Rev. D 84 (2011) 052001.

[5] S. Uehara et al., Phys. Rev. D 86 (2012) 092007.

[6] G. P. Lepage and S. J. Brodsky, Phys. Rev. D 22 (1980) 2157.

[7] V. Braguta, W. Lucha, and D. Melikhov, Phys. Lett. B 661 (2008) 354; I. Balakireva, W. Lucha, and D. Melikhov, J. Phys. G 39 (2012) 055007; Phys. Rev. D 85 (2012) 036006; Phys. Atom. Nucl. 76 (2013) 326.

[8] W. Lucha and D. Melikhov, J. Phys. G 39 (2012) 045003; Phys. Rev. D 86 (2012) 016001.

[9] J. Hořejší and O. V. Teryaev, Z. Phys. C 65 (1995) 691; D. Melikhov and B. Stech, Phys. Rev. Lett. 88 (2002) 151601; D. Melikhov, Eur. Phys. J. direct C4 (2002) 2, arXiv:hep-ph/0110087.

[10] F. Jegerlehner and O. V. Tarasov, Phys. Lett. B 639 (2006) 299; R. S. Pasechnik and O. V. Teryaev, Phys. Rev. D 73 (2006) 034017.

[11] J. Mondejar and K. Melnikov, Phys. Lett. B 718 (2013) 1364.

[12] W. Lucha, D. Melikhov, and S. Simula, Phys. Rev. D 76 (2007) 036002; Phys. Lett. B 657 (2007) 148; Phys. Atom. Nucl. 71 (2008) 1461; Phys. Lett. B 671 (2009) 445; D. Melikhov, Phys. Lett. B 671 (2009) 450.

[13] W. Lucha, D. Melikhov, and S. Simula, Phys. Rev. D 79 (2009) 096011; J. Phys. G 37 (2010) 035003; Phys. Lett. B 687 (2010) 48; Phys. Atom. Nucl. 73 (2010) 1770; J. Phys. G 38 (2011) 105002; Phys. Lett. B 701 (2011) 82; Phys. Rev. D 88 (2013) 056011; W. Lucha, D. Melikhov, H. Sazdjian, and S. Simula, Phys. Rev. D 80 (2009) 114028.

[14] V. A. Nesterenko and A. V. Radyushkin, Phys. Lett. B 115 (1982) 410.

[15] P. Kroll, Eur. Phys. J. C 71 (2011) 1623.

[16] V. V. Anisovich, D. I. Melikhov, and V. A. Nikonov, Phys. Rev. D 55 (1997) 2918; V. V. Anisovich, D. V. Bugg, D. I. Melikhov, and V. A. Nikonov, Phys. Lett. B 404 (1997) 166; T. Feldmann, P. Kroll, and B. Stech, Phys. Rev. D 58 (1998) 114006; Phys. Lett. B 449 (1999) 339.

[17] D. Melikhov and B. Stech, Phys. Rev. D 85 (2012) 051901(R); Phys. Lett. B 718 (2012) 488.

[18] Y. N. Klopot, A. G. Oganesian, and O. V. Teryaev, Phys. Lett. B 695 (2011) 130; Phys. Rev. D 84 (2011) 051901(R); JETP Lett. 94 (2011) 729; Phys. Rev. D 87 (2013) 036013; 88 (2013) 059902(E).

[19] H. L. L. Roberts et al., Phys. Rev. C 82 (2010) 065202; S. J. Brodsky, F.-G. Cao, and G. F. de Téramond, Phys. Rev. D 84 (2011) 033001; 84 (2011) 075012; A. P. Bakulev, S. V. Mikhailov, A. V. Pimikov, and N. G. Stefanis, Phys. Rev. D 84 (2011) 034014; 86 (2012) 031501(R); 87 (2013) 094025; G. F. de Téramond and S. J. Brodsky, arXiv:1203.4025 [hep-ph].

[20] S. S. Agaev, V. M. Braun, N. Offen, and F. A. Porkert, Phys. Rev. D 83 (2011) 054020; 86 (2012) 077504.

[21] P. Masjuan, Phys. Rev. D 86 (2012) 094021; R. Escribano, P. Masjuan, and P. Sanchez-Puertas, arXiv:1307.2061 [hep-ph].

[22] G. M. Huber et al., Jefferson Lab PAC 30 Proposal PR12-06-101 "Measurement of the Charged Pion Form Factor to High $Q^{2, "}(2006)$. 